\title{
Use of a fluoride channel as a new selection marker for fission yeast plasmids and application to fast genome editing with CRISPR/Cas9
}

\author{
Ronan Fernandez ${ }^{1,2}$ and Julien Berro ${ }^{1,2, *}$ \\ ${ }^{1}$ Department of Molecular Biophysics and Biochemistry, Yale University, New Haven, CT 06520, \\ USA \\ ${ }^{2}$ Nanobiology Institute, Yale University, West Haven, CT 06516, USA
}

\begin{abstract}
Fission yeast is a powerful model organism that has provided insights into important cellular processes thanks to the ease of its genome editing by homologous recombination. However, creation of strains with a large number of targeted mutations or containing plasmids has been challenging because only a very small number of selection markers is available in Schizosaccharomyces pombe. In this paper, we identify two fission yeast fluoride exporter channels (Fex1p and Fex2p) and describe the development of a new strategy using Fex1p as a selection marker for transformants in rich media supplemented with fluoride. To our knowledge this is the first positive selection marker identified in $S$. pombe that does not use auxotrophy or drug resistance and that can be used for plasmids transformation or genomic integration in rich media. We illustrate the application of our new marker by significantly accelerating the protocol for genome edition using CRISPR/Cas9 in $S$. pombe.
\end{abstract}

\section{Keywords}

Schizosaccharomyces pombe; fluoride channel; plasmid; CRISPR/Cas9

\section{Introduction}

Schizosaccharomyces pombe is a powerful model system since it is a simple eukaryote that is easy to manipulate. Creation and identification of mutants are facilitated by the existence of selectable markers (Bahler et al., 1998; Grimm et al., 1988; Hentges et al., 2005; Sato et al., 2005) and has allowed fission yeast to be used to make fundamental discoveries in key cellular processes such as cell cycle regulation (Fantes and Nurse, 1977; Nasmyth et al., 1979; Nurse, 1975), cytokinesis (Mulholland et al., 1994; Satterwhite and Pollard, 1992), epigenetics (Nakayama et al., 2001; Volpe et al., 2002) and endocytosis (Arasada and Pollard, 2011; Basu and Chang, 2011; Berro et al., 2010; Sirotkin et al., 2010). Two different

\footnotetext{
*Correspondence to: Julien Berro, Department of Molecular Biophysics and Biochemistry, Yale University, New Haven, CT 06520, USA. julien.berro@yale.edu.

Supporting information: Additional supporting information may be found in the online version of this article at the publisher's website.
} 
classes of positive markers are used in $S$. pombe: auxotrophic markers and drug resistance markers. The disadvantage of auxotrophic markers over drug selection marker is that they cannot be selected on rich media, typically yeast extract (YE), and require plating on minimal media, typically Edinburgh minimal medium (EMM), which allows appearance of new colonies only very slowly (after about a week). In addition, this strategy does not allow for selection of mutants that show limited or no growth on minimal media (Luo et al., 2009; van Slegtenhorst et al., 2004).

A recent advance in $S$. pombe genome edition has been the implementation of the CRISPR/ Cas9 system (Jacobs et al., 2014), which does not require the introduction of any marker in the genome since selection of the plasmid containing Cas9 and the guide RNA is sufficient to ensure high integration efficiency. Yet the protocol developed remains slow since the plasmid contains the auxotrophic selection marker $u r a 4^{+}$, which requires selection on minimal media lacking uracil, therefore increasing the time to recover transformants to about 1 week. This process could be accelerated if one could use a plasmid marker that can be selected on rich media.

Recent studies have shown that a large number of bacteria, archaea and fungi express fluoride export channels protecting them from fluoride toxicity (Baker et al., 2012; Li et al., 2013; Stockbridge et al., 2013). In Saccharomyces cerevisiae, strains lacking both genes encoding these channels (FEX1 and FEX2) are highly sensitive to fluoride, at concentrations as low as $100 \mu \mathrm{M}$ (Smith et al., 2015). In addition, expression of the FEX1 channel from a low copy plasmid is able to fully complement the absence of both fluoride channels and allows budding yeast cells lacking both channels to grow in rich media at high fluoride concentration (up to $5 \mathrm{~mm}$ ), indistinguishably from wild-type strains. These results suggest that fluoride and fluoride channels might be used as a selection marker for plasmid transformation in strains lacking both fluoride channels in $S$. cerevisiae and $S$. pombe grown in rich media.

In this paper, we identify the $S$. pombe homologues of the $S$. cerevisiae fluoride export channels, which we name Fex $1 p$ and Fex $2 p$, and show that they are required to protect cells from fluoride toxicity. We then show that growth of fission yeast strains lacking fex 1 and fex 2 genes in media containing fluoride can be rescued by expressing Fex $1 p$ from a plasmid or at a different genomic locus, and use these properties to repurpose Fex $1 \mathrm{p}$ as a selection marker for plasmid or genomic integration in rich media supplemented with fluoride. Lastly, we use this new marker to improve the throughput of $S$. pombe strain construction using the CRISPR/Cas9 system.

\section{Material and methods}

\section{Strains, media and growth curves}

The fission yeast strains FY527 (h-, his3-D1 leu1-32 ura4-D18 ade6-M216) and FY528 (h+, his3-D1 leu1-32 ura4-D18 ade6-M216) were used as the master strains for most transformations. The strain JBSp224 (h+, his3-D1 leu1-32 ura4-D18 ade6-

M216fex14::natMX6fex24::kanMX6) was created by two successive transformations of FY528 using the standard lithium acetate procedure (Moreno et al., 1991). The strain 
JBSp289 was created by transformation of JBSp224 using the lithium acetate procedure to tag the gene pill with mEGFP and using Fex $1 \mathrm{p}$ as a selection marker. All the strains created and used in this study are listed in Table 1.

Yeast cells were grown in rich medium (YE5S; $0.5 \%$ yeast extract and 3\% dextrose) or Edinburgh minimal medium (EMM5S), both supplemented with adenine, leucine, lysine, histidine and uracil $(0.225 \mathrm{~g} / \mathrm{l}$ each). Media preparation and basic manipulation methods of S. pombe were carried out as described previously (Moreno et al., 1991).

For spot assays, cells were grown overnight and diluted to 0.1 optical density at $595 \mathrm{~nm}$ $\left(\mathrm{OD}_{595}\right)$ and serially diluted in sterile $\mathrm{ddH}_{2} \mathrm{O}$ by $10 \times$ increments. $10 \mu \mathrm{l}$ of cell suspension was spotted onto YE5S plates with varying concentrations of fluoride (NaF) (Sigma, St Louis, MO, USA) and grown at $32{ }^{\circ} \mathrm{C}$ for 3 days prior to imaging. For liquid growth assays, cultures were diluted to $\mathrm{OD}_{595}=0.1$ in 24-well flat-bottom plates (Corning Inc., Corning, NY, USA) containing either YE5S and varying concentrations of fluoride, or EMM5S or YNB5S in a final volume of $1 \mathrm{ml}$. One experiment was performed for each strain and in each condition. The $\mathrm{OD}_{595}$ was measured every 5 min until growth plateaued using a BioTek Synergy 4 plate reader set at 25 or $32{ }^{\circ} \mathrm{C}$ with continual shaking.

Escherichia coli TOP10 cells were used for subcloning polymerase chain reaction (PCR) products and were grown in LB medium.

\section{Cloning and vector design}

Plasmids used in this study are listed in Table 2. The plasmid pMZ374 (Jacobs et al., 2014) was modified by Gibson assembly (Gibson et al., 2009) in order to replace the coding sequence (CDS) of $u r a 4^{+}$by the CDS of $f e x 1^{+}$, which was amplified from the gDNA of the S. pombe strain FY527.

All the guide RNA ( $\operatorname{sgRNA}$ ) vectors used in this study were created by Gibson assembly. The vectors pMZ374 or pJB166 were amplified with JB' 330 (GTTCATCCACGACCTTAACG), JB' 331 (CGTTAAGGTCGTGGATGAAC) and two oligonucleotides designed on the vector around the sgRNA site (TTCTTCGGTACAGGTTATG and GTTTTAGAGCTAGAAATAGCAAG) and also containing a tail for the target sequence to insert.

\section{Genome integration using the pFA6a plasmid and fluoride selection}

The vector pFA6a-mEGFP-kanMX6 was amplified using the primers JB' 801 (GGTTATA CTGAAAAGTATTAGTCAGTACTGACAATAAAAAG) and JB' 802 (GTAAGACTGTGT TAATAGCATGGTTGTTTATGTTCGGATGTG). In parallel, the CDS of the $f e x 1^{+}$gene was amplified from pJB166 using the primers JB' 799

(CACATCCGAACATAAACAACCATGCTATTAACACAGTCTTAC) and JB' 800 (CTTTTTATTGTCAGTACTGACTAATACTTTTCAGTATAACC). The two PCR products were annealed by Gibson cloning to produce pJB193 (pFA6a-mEGFP-fex $1^{+}$). The vector pJB193 was amplified as described (Bahler et al., 1998) so that it contains annealing ends for the gene pill. The PCR product created was used to tag pill with mEGFP in the strain JBSp224 using Fex1p as a selection marker. 


\section{Genome editing using the CRISPR/Cas9 plasmid and fluoride selection}

For genome edition with CRISPR/Cas9, we used strains where both fluoride channels were deleted. Our master strain (JBSp224) was created from FY527 by deleting fex $1^{+}$and fex $2^{+}$. It was transformed using the lithium acetate method (Moreno et al., 1991) with $250 \mathrm{ng}$ of plasmid expressing Fex1p, Cas9 and the sgRNA specific to the region to target (e.g. pJB172) and with $1 \mu \mathrm{g}$ of the PCR fragment used for homology-directed repair, which contains a sequence with the intended editions flanked with sequences homologous to the genomic region around the Cas9 cut. The transformation product was plated on YE5S+1 mM NaF and incubated at $32^{\circ} \mathrm{C}$ for $2-3$ days. Twelve colonies were restreaked on YE5S+1 mu NaF for a further 2-3 days. Colonies appearing after the restreak can be directly screened using colony PCR.

\section{Imaging}

All pictures were taken using a spinning disc confocal microscope based on a TiE inverted (Nikon) instrument equipped with a 100×/1.45 NA objective, a Yokogawa CSU-W1 confocal spinning unit and a Andor iXon Ultra888 EMCCD camera.

\section{Results}

Identification of genes coding for the fluoride export channels Fex1p and Fex2p in $S$. Pombe

Saccharomyces cerevisiae fluoride export channels FEX1 and FEX2 contain 375 amino acids and are $99.2 \%$ identical to each other, with only three different but similar amino acids: V210I, I301V and A351V (supporting information, Figure S1A). We found two homologues of $S$. cerevisiae FEX1 and FEX2 in $S$. pombe: SPAC977.11 and SPBPB 8B6.06c. Similarly to $S$. cerevisiae, the proteins encoded by SPAC977.11 and SPBPB8B6.06c are 100\% identical (supporting information, Figure S1B). However, conservation of the protein sequences between $S$. cerevisiae and $S$. pombe FEX channels is poor (36\%), but several domains contain stretches of amino acid that are conserved or with similar biophysical properties (Figure 1). The high intraspecies and poor interspecies conservation make it difficult to annotate the genes SPAC977.11 and SPBPB8B6.06c. SPAC977.11, being located on chromosome I and SPBPB8B6.06c on chromosome II, we respectively annotated them as fex 1 and $f e x 2$ in $S$. pombe.

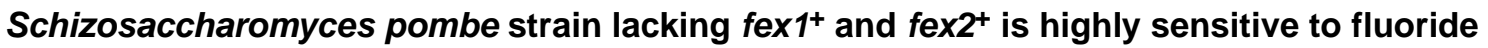

To confirm that Fex1p and Fex2p are indeed fluoride export channels and to test whether the export of fluoride is as essential for survival in $S$. pombe as it is in $S$. cerevisiae, we created an $S$. pombe strain where both $f e x 1$ and $f e x 2$ genes were deleted (JBSp224). Before further analysis, we showed that this newly created strain does not present any obvious morphological phenotype (supporting information, Figure S2). We also showed that it has the same behaviour as a wild-type strain in rich and poor media (Figure 3A, B, blue line; supporting information, Figure S3) and calculated that both strains have a doubling time of about $180 \mathrm{~min}$ in YE5S. This finding suggests that the deletion of both $\mathrm{fex} 1^{+}$and $\mathrm{fex}^{+}$ genes does not have an impact on the physiology of the cell. However, growth of the fex1A 
fex $2 \Delta$ strain was impaired in the presence of as low as $10 \mu \mathrm{m}$ fluoride on solid or liquid rich media (YE5S), whereas strains expressing Fex1p and/or Fex2p showed normal growth in the presence of up to 5 mm fluoride (Figures 2 and 3A, B). Therefore, fluoride is toxic for $S$. pombe even at low concentrations, and specific channels that export fluoride ions are required for normal growth.

\section{Fluoride sensitivity of fex $1 \Delta$ fex $2 \Delta$ can be rescued by expression of $S$. Pombe Fex1p from a plasmid}

We tested whether fluoride sensitivity of the fex $1 \Delta$ fex $2 \Delta$ strain can be rescued by expression of $S$. pombe Fex $1 \mathrm{p}$ from a plasmid. We modified the plasmid pMZ374, originally designed by Jacobs et al. (2014) to perform CRISPR/Cas9 in $S$. pombe, by replacing the ura $4^{+}$auxotrophic allele with $S$. pombe fex $1^{+}$gene, expressed under the ura4 promoter (pJB166; supporting information, Figure S4). We transformed our fex $1 \Delta$ fex $2 \Delta$ strain with either our new plasmid expressing Fex1p (pJB166) or a control plasmid expressing the ura $4^{+}$ allele (pMZ374), and plated transformed cells on YE5S containing $1 \mathrm{~mm}$ fluoride, a concentration that prevents growth of cells lacking both fluoride channels (Figures 2 and 3B). Absence of colonies in our control and the presence of a large number of colonies in our transformation with the plasmid containing $f e x 1^{+}$(pJB166) indicate that Fex $1 \mathrm{p}$ can be expressed from a plasmid and is able to complement the inability of the fex $1 \Delta$ fex $2 \Delta$ strain to export fluoride (supporting information, Figure S5). This result shows that Fex1p expression can be used as a selection marker for transformations into the fex $1 \Delta f \mathrm{fe} 2 \Delta$ strain grown on a rich medium containing fluoride. To our knowledge, Fex1p is the first marker identified in $S$. pombe that does not use auxotrophy or drug resistance and that can be used to select for plasmid in rich media.

\section{Effect of Cas9 on cell growth}

In our initial complementation experiment, we observed that cells transformed with the plasmid expressing Fex1p and Cas9 grew more slowly (Figure 3C) than wild-type cells (Figure 3A) in the presence or absence of fluoride. Since Cas9 is under the control of the strong pTEF promoter in this plasmid, we hypothesized that slower growth could be attributed to the abundant expression of Cas9, as suggested in Jacobs et al. (2014). We engineered a new vector where the Cas9 gene was removed (pJB186; supporting information, Figure S4) and observed that the growth rate of the fex $1 \Delta$ fex $2 \Delta$ strain transformed with this vector was similar to that observed for wild-type cells (Figure 3D). This result confirms that expression of a large amount of Cas9 has some toxic effects for fission yeast growth. A surprising observation is that in the absence of fluoride we do not observe a delay of growth when the cells are transformed with the plasmid expressing Cas9. That result could probably be explained by the fact that in the absence of fluoride there is no selection pressure for cells to keep the plasmid. Cells that have lost the plasmid would then not express Cas9 and grow faster than cells expressing Cas9. Therefore, they would likely have a competitive advantage and rapidly outnumber cells containing the plasmid, which would explain the apparently normal growth curve when no fluoride is present. 


\section{Use of our new fluoride marker (Fex1p) to accelerate genome edition with CRISPR/Cas9}

The search for a non-auxotrophic marker that can be expressed on a plasmid was motivated in the first place by the urge to improve the CRISPR/Cas9 protocol developed by Jacobs et al. (2014) in $S$. pombe. Using a plasmid that expresses Fex1p rather than the ura4 allele makes it possible to recover transformants after 2-3 days on rich media (YE5S) containing 1 mм fluoride, whereas using the original plasmid requires growth on minimal media depleted of uracil (EMM5S-Ura), and colonies appear after 6-7 days. To compare the efficiency of both methods, we used in parallel the original plasmid and our newly developed plasmid to delete the gene coding for the eisosome protein Pillp. In addition to their selection markers, both plasmids expressed the Cas9 protein and a guide RNA with homology with a sequence in the pill gene and containing an NGG PAM sequence (GGACGGCGGTTTGCTGAGGA). In both transformations, we also added a donor DNA created by PCR as the fusion of the 5'UTR (primers used: TCGCCCTTTGGTAAAGTGTT and CAAATA GAAAAAAAGTAAAGAGTTTTTCTTGCAGA AATAGAAATAGATGT) and of the 3'UTR of the pill gene (primers used: ACATCTATTTCTAT TTCTGCAAGAAAAACTCTTTACTTTTTTTC TATTTG and CGAAGCAATTCAATCATAC ACC), which cells could use to repair the double-stranded break induced by Cas9, leading to the deletion of the entire pill gene. As a control, we transformed a wild-type strain (expressing Fex $1 p$ and Fex2p) with the plasmid with the $\mathrm{ura}^{+}$marker (pJB109) and selected on EMM5S-Ura plates. In parallel, we transformed the fex $1 \Delta f e x 2 \Delta$ strain with the plasmid containing the fex $1^{+}$marker and plated on rich medium (YE5S) supplemented with $1 \mathrm{~mm}$ fluoride. After appearance of colonies, we restreaked 12 of them on the appropriate selective medium and screened them to identify pill $\Delta$ strains. In both transformations, four out of 12 colonies were positive, showing that both methods used to edit strains with CRISPR/Cas9 have comparable efficiency ( $33 \%)$. The major advantage of using $\mathrm{fex} 1^{+}$as a selection marker is that it allowed us to identify edited strains in about 5 days, whereas it took more than 11 days to recover strains using the auxotrophic marker ura4 $4^{+}$(Figure 4).

\section{Fex1p can also be used as a selection marker in traditional integration protocols}

We tested whether Fex1p and fluoride can also be used as a marker for traditional genomic integration. As a proof of principle, we modified the pFA6a vector used for C-terminal tagging with mEGFP (Bahler et al., 1998) by replacing the kanMX6 drug resistance gene with the $f e x 1^{+}$gene. We used this plasmid (pJB193) to C-terminally tag with mEGFP the eisosome gene pill using the protocol developed by Bahler et al. (1998). Plating the transformants on rich media supplemented with fluoride produced a large number of colonies whereas the control transformation produced no colonies (supporting information, Figure S6). We confirmed proper integration of the mEGFP and $f e x 1^{+}$cassette by PCR and by imaging the cells with a spinning disc confocal microscope (supporting information, Figure S7). These results show that Fex1p and fluoride can also be used as a selection marker for genomic integration. 


\section{Discussion}

In this study, we overcame the limitations of selection markers currently available in $S$. pombe by repurposing fluoride toxicity and fluoride export channels into a new strategy for selecting transformants. Our new strategy is of particular interest for plasmid transformation since most plasmid markers are auxotrophic and require selection on minimal media, making plasmid transformation slow and poorly efficient in mutants with growth defects. Another advantage of our new strategy is that fluoride is significantly cheaper than classic antibiotics used in $S$. pombe (e.g. 100 times less than Geneticin and 1000 times less than Clonat). An immediate application of this new selection marker has been the dramatic shortening of the protocol of genome edition using the CRISPR/Cas9 system to timeframes comparable to traditional integration methods with antibiotic selection. We expect our new method to have an impact beyond the $S$. pombe community and will be adaptable to other species since genes encoding for fluoride export channels are expressed in most eukaryotes and prokaryotes. The only minor constraint of our new method is that strains that can be edited require all genes coding for fluoride export channels to be removed. We do not expect any issue with these strains since, as far as we have observed, they do not behave any differently than wild-type strains in media containing trace fluoride levels found in doubledistilled water and commercially available reagents.

\section{Supplementary Material}

Refer to Web version on PubMed Central for supplementary material.

\section{Acknowledgments}

The authors thank Kathryn (Katie) Smith, Tetyana (Tanya) Berbasova and Scott Strobel for their insights into fluoride channels and, for technical support, Edward (Eddie) Barbieri and Mikel Zaratiegui for initial help to get started with CRISPR/Cas9 genome edition in yeast. We also thank Neal Ravindra for his help with microscopy. This research was supported in part by a Pilot Grant from the Yale Comprehensive Cancer Center and the American Cancer Society \#IRG-58-012-57 and by the NIH/NIGMS grant R01GM115636.

\section{References}

Arasada R, Pollard TD. Distinct roles for F-BAR proteins Cdc15p and Bzz1p in actin polymerization at sites of endocytosis in fission yeast. Curr Biol. 2011; 21:1450-1459. [PubMed: 21885283]

Bahler J, Wu JQ, Longtine MS, et al. Heterologous modules for efficient and versatile PCR-based gene targeting in Schizosaccharomyces pombe. Yeast. 1998; 14:943-951. [PubMed: 9717240]

Baker JL, Sudarsan N, Weinberg Z, Roth A, Stockbridge RB, Breaker RR. Widespread genetic switches and toxicity resistance proteins for fluoride. Science. 2012; 335:233-235. [PubMed: 22194412]

Basu R, Chang F. Characterization of dip1p reveals a switch in Arp2/3-dependent actin assembly for fission yeast endocytosis. Curr Biol. 2011; 21:905-916. [PubMed: 21620704]

Berro J, Sirotkin V, Pollard TD. Mathematical modeling of endocytic actin patch kinetics in fission yeast: disassembly requires release of actin filament fragments. Mol Biol Cell. 2010; 21:2905-2915. [PubMed: 20587776]

Fantes P, Nurse P. Control of cell size at division in fission yeast by a growth-modulated size control over nuclear division. Exp Cell Res. 1977; 107:377-386. [PubMed: 872891]

Gibson DG, Young L, Chuang RY, Venter JC, Hutchison CA 3rd, Smith HO. Enzymatic assembly of DNA molecules up to several hundred kilobases. Nat Methods. 2009; 6:343-345. [PubMed: 19363495] 
Grimm C, Kohli J, Murray J, Maundrell K. Genetic engineering of Schizosaccharomyces pombe: a system for gene disruption and replacement using the ura4 gene as a selectable marker. Mol Gen Genet. 1988; 215:81-86. [PubMed: 3241624]

Hentges P, Van Driessche B, Tafforeau L, Vandenhaute J, Carr AM. Three novel antibiotic marker cassettes for gene disruption and marker switching in Schizosaccharomyces pombe. Yeast. 2005; 22:1013-1019. [PubMed: 16200533]

Jacobs JZ, Ciccaglione KM, Tournier V, Zaratiegui M. Implementation of the CRISPR-Cas9 system in fission yeast. Nat Commun. 2014; 5:5344. [PubMed: 25352017]

Li S, Smith KD, Davis JH, Gordon PB, Breaker RR, Strobel SA. Eukaryotic resistance to fluoride toxicity mediated by a widespread family of fluoride export proteins. Proc Natl Acad Sci U S A. 2013; 110:19018-19023. [PubMed: 24173035]

Luo J, Matsuo Y, Gulis G, Hinz H, Patton-Vogt J, Marcus S. Phosphatidylethanolamine is required for normal cell morphology and cytokinesis in the fission yeast Schizosaccharomyces pombe. Eukaryot Cell. 2009; 8:790-799. [PubMed: 19286980]

Moreno S, Klar A, Nurse P. Molecular genetic analysis of fission yeast Schizosaccharomyces pombe. Methods Enzymol. 1991; 194:795-823. [PubMed: 2005825]

Mulholland J, Preuss D, Moon A, Wong A, Drubin D, Botstein D. Ultrastructure of the yeast actin cytoskeleton and its association with the plasma membrane. J Cell Biol. 1994; 125:381-391. [PubMed: 8163554]

Nakayama J, Rice JC, Strahl BD, Allis CD, Grewal SI. Role of histone H3 lysine 9 methylation in epigenetic control of heterochromatin assembly. Science. 2001; 292:110-113. [PubMed: 11283354]

Nasmyth K, Nurse P, Fraser RS. The effect of cell mass on the cell cycle timing and duration of Sphase in fission yeast. J Cell Sci. 1979; 39:215-233. [PubMed: 528581]

Nurse P. Genetic control of cell size at cell division in yeast. Nature. 1975; 256:547-551. [PubMed: 1165770]

Sato M, Dhut S, Toda T. New drug-resistant cassettes for gene disruption and epitope tagging in Schizosaccharomyces pombe. Yeast. 2005; 22:583-591. [PubMed: 15942936]

Satterwhite LL, Pollard TD. Cytokinesis. Curr Opin Cell Biol. 1992; 4:43-52. [PubMed: 1313686]

Sirotkin V, Berro J, Macmillan K, Zhao L, Pollard TD. Quantitative analysis of the mechanism of endocytic actin patch assembly and disassembly in fission yeast. Mol Biol Cell. 2010; 21:28942904. [PubMed: 20587778]

Smith KD, Gordon PB, Rivetta A, et al. Yeast Fex1p is a constitutively expressed fluoride channel with functional asymmetry of its two homologous domains. J Biol Chem. 2015; 290:19874-19887. [PubMed: 26055717]

Stockbridge RB, Robertson JL, Kolmakova-Partensky L, Miller C. A family of fluoride-specific ion channels with dual-topology architecture. Elife. 2013; 2:e01084. [PubMed: 23991286]

van Slegtenhorst M, Carr E, Stoyanova R, Kruger WD, Henske EP. Tsc1+ and tsc2+ regulate arginine uptake and metabolism in Schizosaccharomyces pombe. J Biol Chem. 2004; 279:12706-12713. [PubMed: 14718525]

Volpe TA, Kidner C, Hall IM, Teng G, Grewal SI, Martienssen RA. Regulation of heterochromatic silencing and histone H3 lysine-9 methylation by RNAi. Science. 2002; 297:1833-1837. [PubMed: 12193640] 


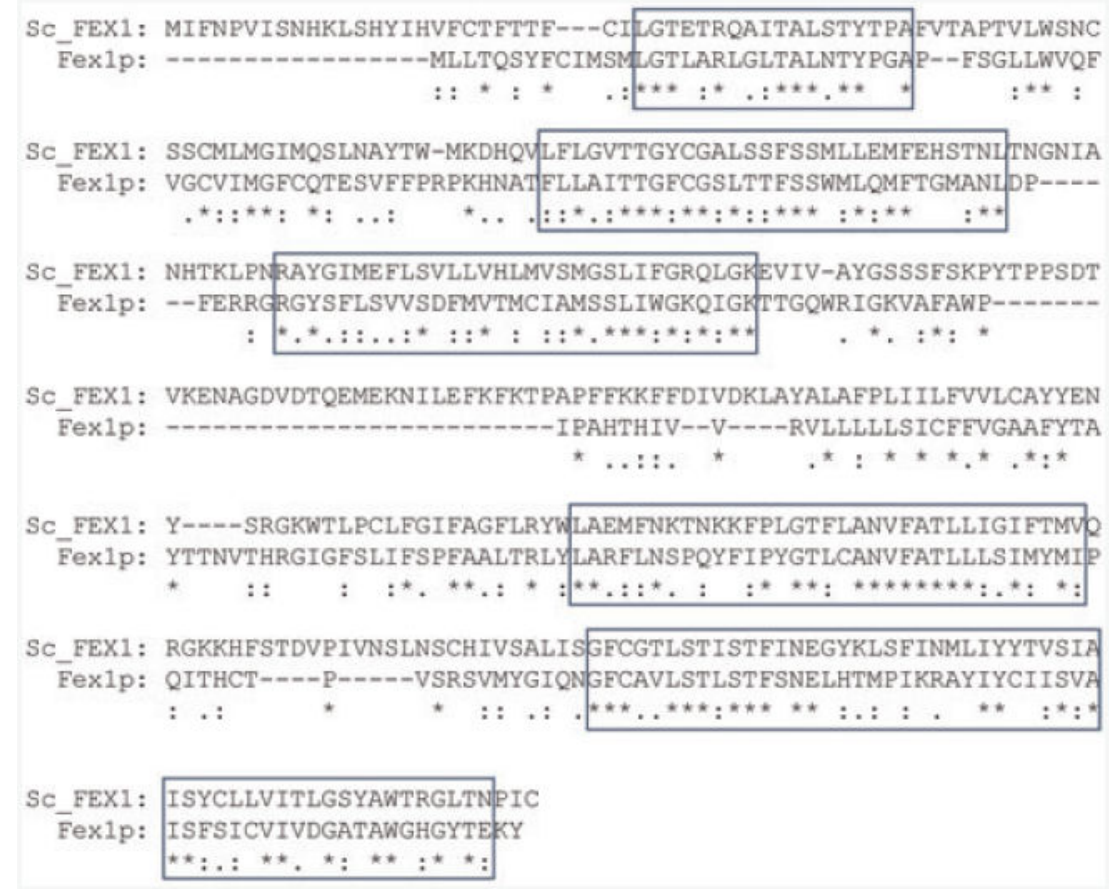

Figure 1.

The fluoride channels from S. pombe (Fex $1 p$ and Fex2p) and S. cerevisiae (FEX1 and FEX2) share only $36 \%$ identical amino acids but have several conserved regions that contain amino acids with similar properties (boxes). See also Figure S1. The alignment was performed with Clustal Omega. Symbols: asterisk (*), fully conserved residues; colon (:), residues with strong similar properties; point (.), residues with weak similar properties 


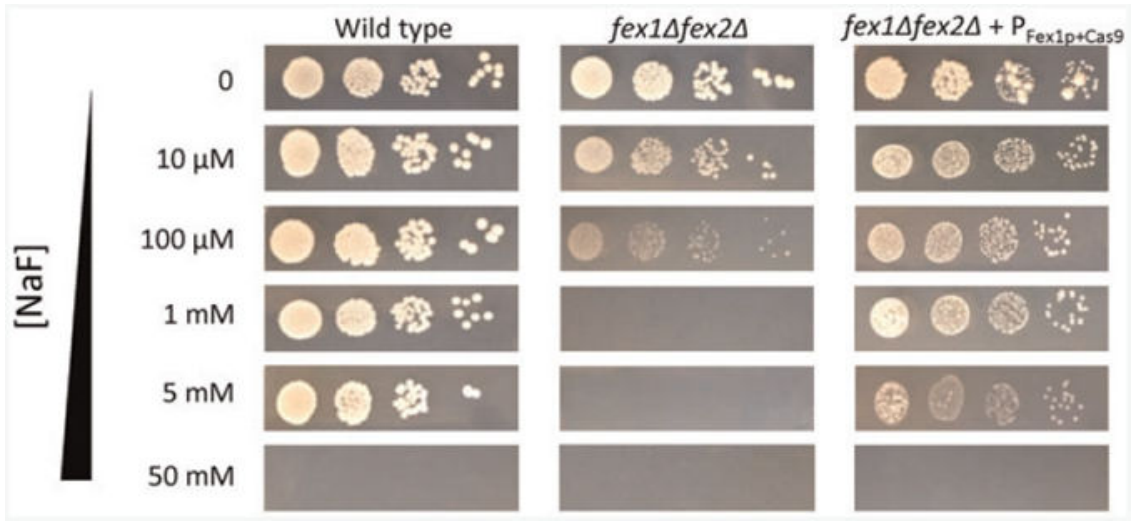

Figure 2.

Spot assays showing that growth of wild-type strains on solid media is insensitive to fluoride up to $5 \mathrm{~mm}$, and growth of the fex $1 \Delta$ fex $2 \Delta$ double mutant is impaired starting at $10 \mu \mathrm{m}$ but can be rescued by expression of Fex $1 p$ from a plasmid (also expressing Cas9) 

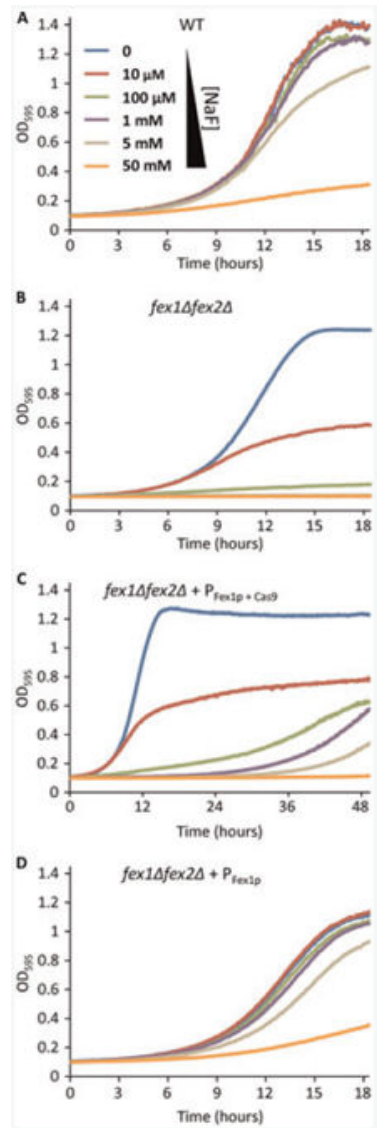

Figure 3.

Growth of $S$. pombe in the presence of different concentrations of fluoride. (A) Growth of the wild-type strain in liquid media is insensitive to fluoride concentrations up to $5 \mathrm{~mm}$. (B) Growth of the fex $1 \Delta f e x 2 \Delta$ strain is similar to wild-type in the absence of fluoride but its growth is impaired for fluoride concentration higher than $10 \mu \mathrm{M}$. (C) Growth of the fex $1 \Delta f e x 2 \Delta$ strain on fluoride can be rescued by the expression of Fex1p on a plasmid. Note that the time-scale for this figure is longer than for other figures because the expression of Cas9 from the plasmid delays the growth of $S$. pombe. (D) The absence of expression of Cas9 allows the growth of $S$. pombe to be rescued with growth curves similar to wild-type 


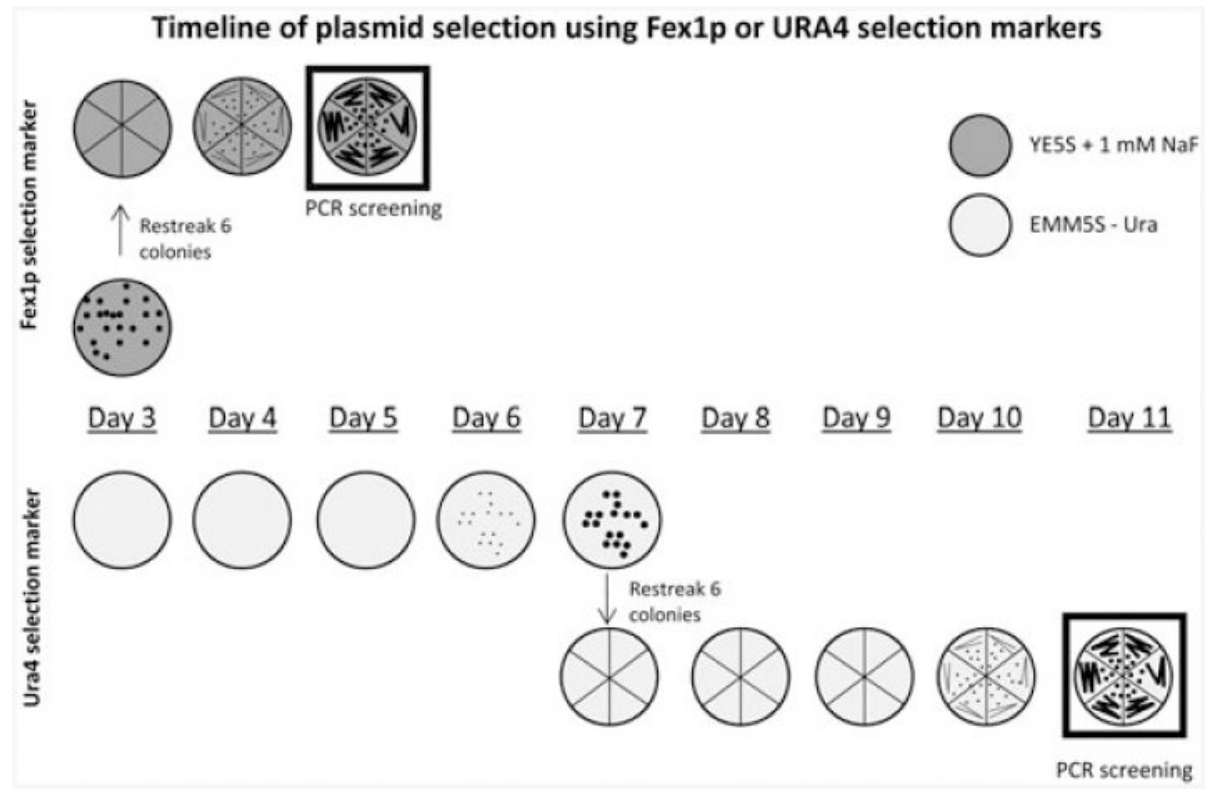

Figure 4.

Comparison of timelines using auxotrophic or fluoride channel markers, as observed in genome edition using CRISPR/Cas9. (Top) Using a Fex1p-expressing plasmid and selection on rich media with fluoride allows the screening for positive colonies 5 days after the initial transformation. (Bottom) Using a ura $4^{+}$-expressing plasmid and minimal media lacking uracil allows the screening for positive colonies after at least 11 days 
Table 1

Strains used in this study

\begin{tabular}{lll}
\hline Name & Genotype & Reference \\
\hline FY527 & ade6-M216 his3-D1 leu1-32 ura4-D18 & S. Forsburg \\
JBSp217 & pil14 ade6-M216 his3-D1 leu1-32 ura4-D18 & This study \\
JBSp224 & fex14::natMX6 fex24::kanMX6 ade6-M216 his3-D1 leu1-32 ura4-D18 & This study \\
JBSp289 & pil1-mEGFP-feX 1+ fex14::natMX6 fex24::kanMX6 ade6-M216 his3-D1 leu1-32 ura4-D18 & This study \\
\hline
\end{tabular}




\section{Plasmids used in this study}

\section{Table 2}

\begin{tabular}{|c|c|}
\hline Name & Description \\
\hline pJB109 & pMZ374 with a gRNA to target pill \\
\hline pJB166 & pMZ374 with ura $4^{4}$ replaced by $f e x l^{+}$ \\
\hline pJB172 & pJB166 with a gRNA to target pill \\
\hline pJB186 & pJB166 with Cas9 deleted \\
\hline pJB193 & pFA6a-mEGFP- $f e x 1^{+}$ \\
\hline
\end{tabular}

